\title{
Sequence-based comparative study of classical swine fever virus genogroup 2.2 isolate with pestivirus reference strains
}

\author{
Ravi Kumar ${ }^{1,2}$, Kaushal Kishor Rajak ${ }^{1}$, Tribhuwan Chandra ${ }^{2}$, Dhanavelu Muthuchelvan ${ }^{1}$, Arpit Saxena ${ }^{1}$, \\ Dheeraj Chaudhary ${ }^{1}$, Ajay Kumar ${ }^{1}$ and Awadh Bihari Pandey ${ }^{1}$
}

1. Division of Virology, Indian Veterinary Research Institute, Mukteswar, Nainital, Uttarakhand, India;

2. Department of Biotechnology, Graphic Era University, Dehradun, Uttarakhand, India.

Corresponding author: Kaushal Kishor Rajak, e-mail: kaushalvirol@gmail.com,

RK: kravi2509@gmail.com, TC: tribhuwan.chandra@gmail.com, DM: drchelva@gmail.com, AS: arpitsxena@gmail.com,

DC: dheeraj.int600@gmail.com, AK: dr.ajayyadav@hotmail.com, ABP: abpandey58@rediffmail.com

Received: 20-05-2015, Revised: 31-07-2015, Accepted: 08-08-2015, Published online: 10-09-2015

doi: 10.14202/vetworld.2015.1059-1062 How to cite this article: KumarR, Rajak KK, Chandra T, Muthuchelvan D, Saxena A, Chaudhary D, Kumar A, Pandey AB (2015) Sequence-based comparative study of classical swine fever virus genogroup 2.2 isolate with pestivirus reference strains, Veterinary World 8(9):1059-1062.

\begin{abstract}
Aim: This study was undertaken with the aim to compare and establish the genetic relatedness between classical swine fever virus (CSFV) genogroup 2.2 isolate and pestivirus reference strains.

Materials and Methods: The available complete genome sequences of CSFV/IND/UK/LAL-290 strain and other pestivirus reference strains were retrieved from GenBank. The complete genome sequence, complete open reading frame, 5' and 3' non-coding region (NCR) sequences were analyzed and compared with reference pestiviruses strains. Clustal W model in MegAlign program of Lasergene 6.0 software was used for analysis of genetic heterogeneity. Phylogenetic analysis was carried out using MEGA 6.06 software package.

Results: The complete genome sequence alignment of CSFV/IND/UK/LAL-290 isolate and reference pestivirus strains showed 58.9-72\% identities at the nucleotide level and 50.3-76.9\% at amino acid level. Sequence homology of 5' and 3' NCRs was found to be $64.1-82.3 \%$ and $22.9-71.4 \%$, respectively. In phylogenetic analysis, overall tree topology was found similar irrespective of sequences used in this study; however, whole genome phylogeny of pestivirus formed two main clusters, which further distinguished into the monophyletic clade of each pestivirus species. CSFV/IND/UK/LAL-290 isolate placed with the CSFV Eystrup strain in the same clade with close proximity to border disease virus and Aydin strains.

Conclusion: CSFV/IND/UK/LAL-290 exhibited the analogous genomic organization to those of all reference pestivirus strains. Based on sequence identity and phylogenetic analysis, the isolate showed close homology to Aydin/04-TR virus and distantly related to Bungowannah virus.
\end{abstract}

Keywords: classical swine fever virus, genogroup, genome, pestivirus, phylogenetic tree, sequence.

\section{Introduction}

The genus pestivirus of the family Flaviviridae comprised of bovine viral diarrhea virus-1 (BVDV-1), BVDV-2, classical swine fever virus (CSFV), and border disease virus (BDV) [1]. Apart from these four established species, various tentative species also detected viz. pestivirus of giraffe [2], BVDV-3 or atypical bovine pestiviruses ("HoBi"-like viruses) [3,4], pronghorn virus [5], Bungowannah virus [6] and Tunisian sheep virus (TSV) (previously termed "Tunisian isolates"), and Turkey sheep virus [7]. Further, BVDV-1, BVDV-2, BDV, and CSFV have been characterized into genotypes, subtypes, and subgenotypes; BVDV-1 and BVDV-2 are having 17 and 3 subtypes, respectively; BDV and CSFV is comprised of 7 and 3 genotypes, respectively, CSFV three genotypes can be further divided into three or four subgenotypes [8].

Pestiviruses have single-stranded, positive-sense RNA genome of approximately 12.3-12.7 kilobases

Copyright: The authors. This article is an open access article licensed under the terms of the Creative Commons Attributin License (http:// creative commons.org/licenses/by/2.0) which permits unrestricted use, distribution and reproduction in any medium, provided the work is properly cited. (kb) that contains only one open reading frame (ORF). The ORF translated into a polyprotein about 3,900 amino acids (aa) flanked by highly conserved non-coding regions (NCRs) at 5'nd 3'ends. The ORF encodes a polyprotein that is co- and post-translationally processed into twelve structural and non-structural proteins. These proteins arranged from 5' to 3' ends are: N-terminal protein (Npro), capsid protein (C), envelope glycoprotein (Erns, E1 and E2), protein $7(\mathrm{p} 7)$, and the non-structural proteins (NS) NS2, NS3, NS4A, NS4B, NS5A, and NS5B [1].

Molecular epidemiology based on complete nucleotide sequence data has broadened the scope for genetic heterogeneity and phylogenetic grouping of isolates existing in field condition. The genetic relatedness of pestivirus isolates, including their relationship to the type viruses of the species, is one of the important parameters for classification of pestiviruses [9]. During the past decade, much progress on the molecular characterization of pestiviruses has been achieved. Although, few isolates have been sequenced completely, and comparative studies on full genome sequence are scanty [10-13]. Generation of complete genome sequence data will help in better 
understanding of the molecular biology of any virus both at structural and functional level. There is no report where CSFV has been compared with other members of pestiviruses, although reverse studies are various [8,13-16]. Recently, full genome sequencing of CSFV genogroup 2.2 isolate, CSFV/IND/UK/LAL290 has been done [17]. To compare CSFV/IND/UK/ LAL-290 isolate with other members of pestiviruses, the present study was undertaken.

\section{Materials and Methods}

\section{Ethical approval}

Ethical approval was not required for this type of study as live animal is not used anywhere in this study.

\section{Sequence Retrieval and Analysis}

Sequences used in this study were retrieved from GenBank (http://www.ncbi.nlm.nih.gov/genbank/) on 2 April 2015 (Table-1). Different sequences like the complete genome complete ORF, 5' and 3' NCR were aligned with respective sequences of reference pestiviruses strain. Sequence analysis was carried out by Clustal W model in MegAlign program of Lasergene 6.0 software (DNASTAR Inc., Madison, WI, USA).

\section{Phylogenetic Analysis}

Phylogenetic analysis was performed using MEGA 6.06 software package [18]. The evolutionary history was inferred using the maximum likelihood method, and the tree topologies were evaluated using 1000 replicates (bootstrap value) of the data set. Best DNA/protein model for construction of phylogenetic tree was selected from model test program integrated with MEGA 6.06 software. Trees based on $5^{\prime}$ and $3^{\prime}$ NCRs sequence were constructed using Kimura $2(\mathrm{G})$ and Tamura $3(\mathrm{G})$ parameters, respectively. Whereas, trees of the complete genome and complete ORF sequence were constructed by general time reversible parameters having gamma distribution with invariable sites $(\mathrm{G}+\mathrm{I})$.

\section{Results and Discussion}

Like other RNA viruses, pestiviruses also exhibit genetic heterogeneity which has been supported by several workers [10-16]. Comparative sequence analysis of CSFV/IND/UK/LAL-290 exhibited analogous genomic organization to those from reference pestivirus strains. CSFV isolate under study showed genome length of 12,297 nucleotides (nt), including a 373-nt 5' untranslated region (UTR), an 11,697-nt ORF encoding a 3,898-amino-acid-long polyprotein, and a 227 -nt 3' UTR [17]. The average sequence length of complete genome, ORF (both nucleotides and amino acids), 5 ' and 3' NCRs of reference pestivirus strains were found to be 12255-12656 bp, 11688-11970 bp (38953989 -aa), 355-399 bp and 206-500 bp, respectively (Table-1). Upon complete genome analysis, BVDV2/ C413 strain exhibited the smallest genome (12255 bp); while Bungowannah strain has the largest genome (12656 bp) amongst reference pestivirus strains. BDV/ X818 and pestivirus Aydin/04-TR shared the smallest ORF with 3895 aa while, pestivirus Giraffe genome showed the largest ORF with 3989 aa.

The sequence alignment of CSFV/IND/UK/ LAL-290 isolate with reference pestivirus strains of complete genome level showed 58.9-72\% identities at the nucleotide level and $50.3-76.9 \%$ at amino acid level. Further 5' and 3' NCRs of the isolate showed $64.1-82.3 \%$ and $22.9-71.4 \%$ sequence identity, respectively as compared to other pestiviruses. The 5'NCR of present CSFV isolate was also found to be highly conserved than 3'NCR like other pestiviruses. Among the genus of family Flaviviridae, pestivirus has relatively long 5' NCR. The 5' NCR of the present isolate was most similar to that of other members of CSFV and BVD with regard to length and sequence, which corroborate the finding of Becher et al. [13]. The 3' NCR of CSFV/IND/UK/LAL-290 genome showed considerable heterogeneity in size. This is similar to the earlier report for BDV, BVDV-1, and other CSFV strains. For BVDV-1 strains (NADL and SD-1) and for most CSFV strains, the 3' end has been reported to be approximately 226 nucleotides long, while those of BVDV-1 strain Osloss and BVDV-2 strain 890 comprised only 185 and 206 nucleotides, respectively [13]. The 3' NCR of CSFV C-strain consists of 241 nucleotides. Surprisingly, the 3' NCR of present

Table-1: Heterogeneity and percent identity of CSFV/IND/UK/LAL-290 with reference to pestivirus strains.

\begin{tabular}{|c|c|c|c|c|c|c|c|c|c|c|c|}
\hline \multirow{3}{*}{$\begin{array}{l}\text { Strains } \\
\text { CSFV/IND/UK/LAL-290* }\end{array}$} & \multirow{3}{*}{$\begin{array}{c}\begin{array}{c}\text { Accession } \\
\text { no. }\end{array} \\
\text { KC_851953 }\end{array}$} & \multirow{2}{*}{\multicolumn{2}{|c|}{$\begin{array}{l}\text { Complete genome } \\
\text { (nucleotide) }(\%)\end{array}$}} & \multicolumn{4}{|c|}{ ORF (\%) } & \multicolumn{4}{|c|}{ NCRs (\%) } \\
\hline & & & & \multicolumn{2}{|c|}{ Nucleotide } & \multicolumn{2}{|c|}{ Amino acid } & \multicolumn{2}{|c|}{ 5' NCR } & \multicolumn{2}{|c|}{ 3' NCR } \\
\hline & & 12297 bp & - & 11697 bp & - & 3898 aa & - & $373 \mathrm{bp}$ & - & $227 \mathrm{bp}$ & - \\
\hline CSFV/Eystrup** & NC_002657 & $12301 \mathrm{bp}$ & 84.1 & 11697 bp & 83.7 & 3898 aа & 89.7 & 373 bp & 93 & $231 b p$ & 85 \\
\hline $\mathrm{BDV} / \mathrm{X} 818$ & NC_003679 & 12333 bp & & 116 & 67.3 & & & 372 bp & 76.1 & 273 bp & 31.2 \\
\hline NADL & & $12573 \mathrm{bp}$ & 66.8 & 1196 & 67 & 3988 aa & 67.2 & $385 \mathrm{bp}$ & 67.3 & $221 \mathrm{bp}$ & 42.7 \\
\hline BVDV2/C413 & $=002032$ & $12255 \mathrm{bp}$ & 68.1 & 11694 bp & 67.9 & 3897 aa & 69.8 & $355 \mathrm{bp}$ & 74.3 & 206 bp & 58.6 \\
\hline BVDV3/Th/04 KhonKaen & E_012812 & $12337 \mathrm{bp}$ & 68.1 & $11700 \mathrm{bp}$ & 68 & 3899 aa & 69.7 & $383 \mathrm{bp}$ & 77.5 & $254 \mathrm{bp}$ & 36.1 \\
\hline Pestivirus Giraffe & C_003678 & 12602 bp & 72 & $11970 \mathrm{bp}$ & 71.6 & 3989 aa & 76.9 & 382 bp & 82.3 & $250 \mathrm{bp}$ & 71.4 \\
\hline Pestivirus Aydin/04-TR & NC_018713 & 12292 bp & 71.2 & $11688 \mathrm{bp}$ & 70.8 & 3895 aа & 75.2 & 277 bp & 79.6 & 227 bp & 55.5 \\
\hline Pestivirus pronghorn antelope & NC_024018 & 12273 bp & 58.9 & 11694 bp & 59.6 & 3897 aа & 50.3 & 369 bp & 64.1 & $210 \mathrm{bp}$ & 22.9 \\
\hline Pestivirus Bungowannah & NC_023176 & 12656 bp & 62 & 11757 bp & 61.1 & 3918 aа & 56.4 & $399 \mathrm{bp}$ & 68.8 & $500 \mathrm{bp}$ & 51.4 \\
\hline
\end{tabular}

*CSFV isolate under study, **CSFV reference strain, $\mathrm{bp}=$ base pair (nucleotide), aa=amino acid, \%=percent identity, $\mathrm{BVDV}=$ Bovine viral diarrhea virus, $\mathrm{ORF}=$ Open reading frame, $\mathrm{NCR}=$ Non-coding region, $\mathrm{CSFV}=\mathrm{Classical}$ swine fever virus, $\mathrm{BDV}=$ Border disease virus 
isolate consisted of 227 nucleotides which were similar to pestivirus Aydin/04-TR.

The percent identity of CSFV/IND/UK/LAL290 with reference pestivirus strains is summarized in Table-1; wherein it showed close homology with Aydin/04-TR virus and distant relation with Bungowannah virus. When the whole genome of CSFV/IND/UK/LAL-290 isolate compared with other submitted CSFV strains; it showed 82.0-91.1\% identities at the nucleotide level and $87.9-92.5 \%$ at amino acid level [17]. Comparison with CSFV reference strain Eystrup (genogroup 1.1 virus) revealed 84.1\% and $89.7 \%$ identity at nucleotide and amino acid level, respectively (Table-1).

Phylogenetic analysis of pestiviruses is generally carried out based on sequences from the 5' UTR/ NCR, Npro and E2 regions [5,19] or, less frequently, the Npro, E2 and NS3 regions $[3,6]$. The topology or topography of trees based on the complete genome (Figure-1a), complete ORF (Figure-1b) and 5' NCR (Figure-1c) sequences were very similar to each of them in comparison to 3' NCR.

The whole genome phylogeny of pestivirus formed two main clusters, which further distinguished into a monophyletic clade of each pestivirus species. CSFV/IND/UK/LAL-290 isolate placed with the CSFV Eystrup strains in the same clade with close proximity to BDV and Aydin strain (Figure-1a). Pestiviruses of bovine (BVDV-1, BVDV-2, and BVDV-3) were clustered separately from CSFV isolates whereas, Bungowannah and pronghorn antelope formed an independent clade. Results of the present study support the findings of Liu et al. [20], but clustering of pestivirus of giraffe was found to be in contrary to them. Pestivirus of giraffe was clustered with CSFV and BDV. The plausible reasons for different tree position of pestivirus of the giraffe could be the use of the different genomic region for making the phylogenetic tree. Liu et al. [20] used combined gene sequences of 5' UTR, Npro and E2 regions for the tree; whereas whole genome was considered in the present study, which has given better resolution of the tree.

In 3' NCR based tree, all pestivirus strains changed their clustering pattern barring strains of the swine (CSFV strains) and ovine (BDV and Aydin strain) origin (Figure-1d). This could be due to high heterogeneity of the region. Study on phylogenetic analysis using this region has not been done so far where all pestiviruses were taken into consideration. To the best of our knowledge, this is the first report of its own kind where attempts were made to analyze all the reference pestiviruses, however, further study is warranted.

Based on sequence analysis using different region and strategies, different workers have proposed their classification of pestiviruses. Analysis of the genetic and antigenic similarities, Becher et al. [19] have suggested seven major antigenic groups of novel pestivirus genotype corresponding to BVDV-1, BVDV-2, CSFV, BDV-1, BDV-2, BDV-3, and "giraffe." Later on, isolation and identification of antelope, Bungowannah, and Tunisian sheep virus lead to a new proposal of classification. A new proposal is presented for the classification of pestiviruses into nine species: BVDV-1, BVDV-2, BVDV-3 (atypical bovine pestiviruses), pestivirus of giraffe, CSFV, BDV, Tunisian sheep virus (Aydin/04-TR), Antelope, and Bungowannah [20]. Findings of this study also proposed a classification of novel pestiviruses into nine species similar to Liu et al. [20]; however, clustering pattern in phylogeny was found to be different.

\section{Conclusion}

In the present study, it was observed that CSFV/ IND/UK/LAL-290 showed a comparable genomic

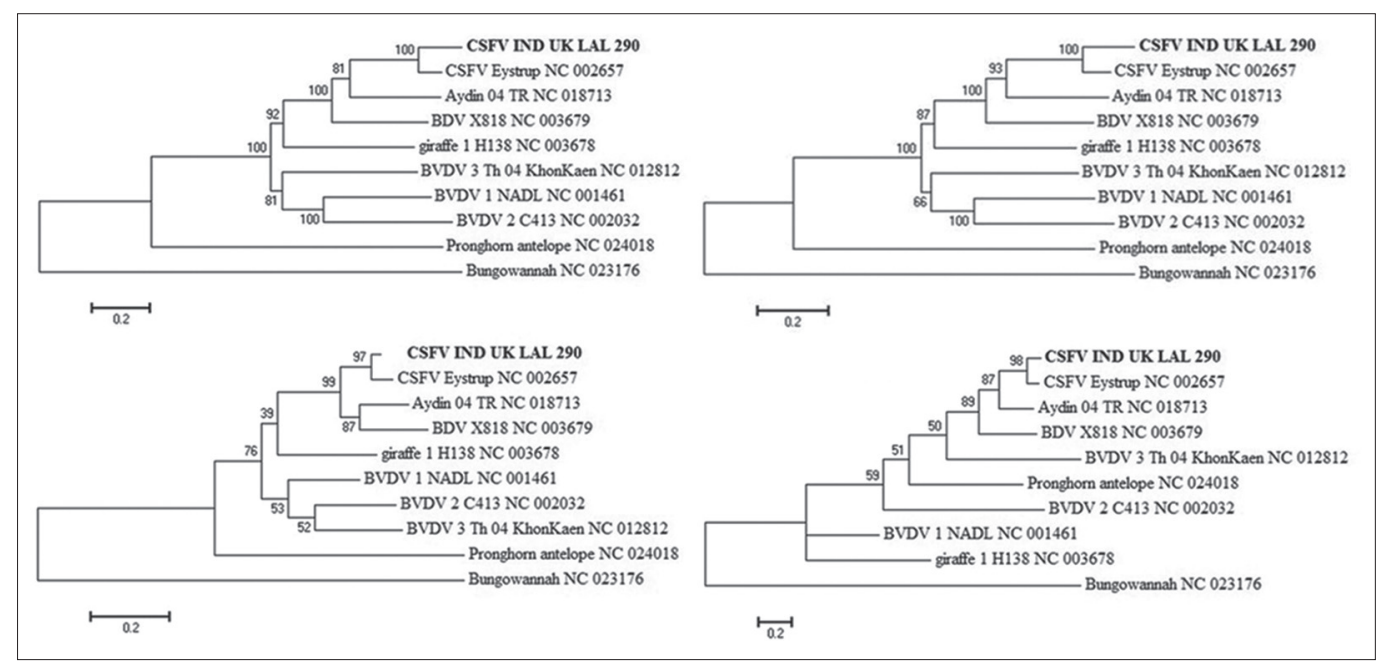

Figure-1: Maximum likelihood (ML) trees depicting phylogenetic relationship of classical swine fever virus/IND/UK/LAL290 isolate among pestivirus reference strains at, (a) complete genome and (b) open reading frame (ORF) nucleotide sequence level with general time reversible (GTR+G+I) model; (c) $5^{\prime}$ NCR with Kimura 2 (K2+G) model, and (d) 3' NCR with Tamura $3(\mathrm{~T} 92+\mathrm{G})$ model. The bootstrap consensus was inferred from 1,000 replicates to represent the evolutionary history of the taxa analyzed, and branch lengths were indicative of genetic distances between the sequences. 
organization with that of other pestiviruses. The isolate was closely related to Aydin/04-TR virus and distantly with Bungowannah virus. Phylogenetic analysis revealed that BVDV-1, BVDV-2, BVDV-3 sharing a common ancestor, while Bungowannah and pronghorn antelope formed a single separate clade in all the trees. In contrary to the previous findings, pestivirus of giraffe was clustered with CSFV and BDV. Various researchers have classified the pestiviruses in independent manner and availability of the virus sequences. Proposal of nine species in novel pestiviruses also held true in the present study; though, grouping of viruses was different. This study provides a guideline for the classification of newly detected pestiviruses and has a potential application in genetic analysis of other related viruses in future.

\section{Authors' Contributions}

RK, KKR, and TC conceptualized the work. RK, $\mathrm{DM}, \mathrm{DC}, \mathrm{AS}$ and AK carried out the work and bioinformatics part. KKR, ABD, and RK drafted the manuscript. KKR, DM, ABP, and TC reviewed and edited the manuscript. All authors read the manuscript and approved the final manuscript.

\section{Acknowledgments}

The authors would like to thank the Director, Indian Veterinary Research Institute, Izatnagar, Uttar Pradesh and Department of Biotechnology (DBT), Ministry of Science and Technology, Government of India, New Delhi for providing fund and facilities to carry out the work.

\section{Competing Interests} interests.

The authors declare that they have no competing

\section{References}

1. Simmonds, P., Becher, P., Collet, M.S., Gould, E.A., Heinz, F.X., Meyers, G., Monath, T., Pletnev, A., Rice, C.M., Stiansny, K., Thiel, H.J., Weiner, A. and Bukhet, J. (2011). Flaviviridae. In: King, A.M.Q., Adams, M.J., Carstens, E.B. and Lefkowitz, E.J., editors. Virus Taxonomy: Ninth Report of the International Committee on Taxonomy of Viruses. Academic Press, San Diego. p1003-1020.

2. Avalos-Ramirez, R., Orlich, M., Thiel, H.J. and Becher, P. (2001). Evidence for the presence of two novel Pestivirus species. Virology, 286: 456-465.

3. Schirrmeier, H., Strebelow, G., Depner, K., Hoffmann, B. and Beer, M. (2004). Genetic and antigenic characterization of an atypical pestivirus isolate, a putative member of a novel pestivirus species. J. Gen. Virol., 85: 3647-3652.

4. Bauermann, F.V., Ridpath, J.F., Weiblen, R. and Flores, E.F. (2013). HoBi-like viruses: An emerging group of pestiviruses. J. Vet. Diagn. Investig., 25: 6-15.

5. Vilcek, S., Ridpath, J.F., Van Campen, H., Cavender, J.L. and Warg, J. (2005). Characterization of a novel pestivirus originating from a pronghorn antelope. Virus Res., 108: 187-193.

6. Kirkland, P.D., Frost, M.J., Finlaison, D.S., King, K.R., Ridpath, J.F. and $\mathrm{Gu}, \mathrm{X}$. (2007). Identification of a novel virus in pigs - Bungowannah virus: A possible new species of pestivirus. Virus Res., 129: 26-34.

7. Oguzoglu, T.C., Tan, M.T., Toplu, N., Demir, A.B., BilgeDagalp, S., Karaoglu, T., Ozkul, A., Alkan, F., Burgu, I., Haas, L. and Greiser-Wilke, I. (2009). Border disease virus (BDV) infections of small ruminants in Turkey: A new BDV subgroup? Vet. Microbiol., 135: 374-379.

8. Weber, M.N., Streck, A.F., Silveira, S., Mósena, A.C.S., da Silva, M.S. and Canal, C.W. (2015). Homologous recombination in pestiviruses: Identification of three putative novel events between different subtypes/genogroups. Infect. Genet. Evol., 30: 219-224.

9. Heinz, F.X., Collett, M.S., Purcell, R.H., Gould, E.A., Howard, C.R., Houghton, M., Moormann, R.J.M., Rice, C.M. and Thiel, H.J., (2000). Family Flaviviridae. Academic Press, San Diego, CA.

10. Vilcek, S., Leskova, V., Meyer, D., Postel, A. and Becher, P. (2014). Molecular characterization of border disease virus strain Aveyron. Vet. Microbiol., 171: 87-92.

11. Mao, L., Li, W., Zhang, W., Yang, L. and Jiang, J. (2012). Genome sequence of a novel Hobi-like pestivirus in China. J. Virol., 86: 12444.

12. Gupta, P.K., Saini, M., Dahiya, S.S., Patel, C.L., Sonwane, A.A., Rai, D.V. and Pandey, K.D. (2011). Molecular characterization of lapinized classical Swine Fever vaccine strain by full-length genome sequencing and analysis. Anim. Biotechnol., 22: 111-117.

13. Becher, P., Orlich, M. and Thiel, H.J. (1998). Complete genomic sequence of border disease virus, a pestivirus from sheep. J. Virol., 72: 5165-5173.

14. Becher, P., Schmeiser, S., Oguzoglu, T.C. and Postel, A. (2012). Complete genome sequence of a novel pestivirus from sheep. J. Virol., 86: 11412.

15. Giangaspero, M. and Harasawa, R. (2011). Species characterization in the genus Pestivirus according to palindromic nucleotide substitutions in the 5 '-untranslated region. $J$. Virol. Methods, 174: 166-172.

16. Postel, A., Schmeiser, S., Oguzoglu, T.C., Indenbirken, D., Alawi, M., Fischer, N., Grundhoff, A. and Becher, P. (2015). Close relationship of ruminant pestiviruses and classical swine fever virus. Emerg. Infect. Dis., 21: 668-672.

17. Kumar, R., Rajak, K.K., Chandra, T., Thapliyal, A., Muthuchelvan, D., Sudhakar, S.B., Sharma, K., Saxena, A., Raut, S.D., Singh, V.K., Ahmad, Z., Kumar, A., Chaudhary, D., Singh, R.K. and Pandey, A.B. (2014). Whole-genome sequence of a classical swine fever virus isolated from the Uttarakhand state of India. Genome Announc., 2(3): e00371-14.

18. Tamura, K., Stecher, G., Peterson, D., Filipski, A. and Kumar, S. (2013). MEGA6: Molecular evolutionary genetics analysis version 6.0. Mol. Biol. Evol., 30: 2725-2729.

19. Becher, P., Raminez, R.A., Orlich, M., Rosales, S.C., Konig, M., Schweizer, M., Stalder, H., Schirrmeier, H. and Thiel, H.J. (2003). Genetic and antigenic characterization of novel pestivirus genotypes: Implication for classification. Virology, 311: 96-104.

20. Liu, L., Xia, H., Wahlberg, N., Belák, S. and Baule, C. (2009). Phylogeny, classification and evolutionary insights into pestiviruses. Virology, 385: 351-357. 